\title{
NSAID-induced gastric ulcers: Exploring the silent dilemma
}

\author{
RICHARD H HUNT, FRCP, FACG, FRCPC
}

$\mathrm{T}$ his special issue of The Canadian Joumal of Gastroenterology is devoted to the proceedings of a satellite symposium held at the time of the Fourth International Course in Therapeutic Endoscopy which took place in Toronto in October 1989. The symposium, sponsored by Searle Canada, took advantage of the large gathering of Canadian and overseas gastroenterologists to discuss some of the current controversies surrounding NSAIDs and gastroduodenal damage. The presentations, given by an international panel of experts, focused on the following issues: the risk of developing gastric and duodenal lesions and the extent of this risk with regard to complications of bleeding and perforation; how symptoms should be managed and ulcers treated depending upon their location; and prophylaxis, particularly regarding the problems related to identifying who should receive prophylactic treatment and with what medication.

Studies reporting symptoms in patients taking NSAIDs show a considerable range from 10 to $60 \%$ with a mean of about one-third of patients experiencing problems at three months. But do all symptomatic patients require investigation and treatment? In many cases severity will decline over the first few weeks as tolerance develops, and loss of symptoms may coincide with a change in the choice of NSAID which is often credited with the improvement. Other alternatives include reducing the dose or giving the

Dr Hunt is the Head of the Division of Gastroenterology, Department of Medicine, McMaster University, Hamilton, Ontario

Correspondence: Dr Richard H Hunt, McMaster University Medical Centre, Hamilton, Ontario L8N 325. Telephone

(416)521-2100 ext 6404
NSAID with food or antacids, although these approaches have not been well studied. Few treatments are effective in controlling the symptoms associated with NSAID ingestion, although some have suggested that the $\mathrm{H}_{2}$ receptor antagonists are effective (1) and that sucralfate may be helpful (2).

At what point should investigation be undertaken? It seems wise to undertake full investigation with endoscopy for patients whose symptoms persist, or who experience anemia, weight loss or other symptoms. However, it is clear that symptoms are a poor predictor of the presence of an ulcer or subsequent complications. Endoscopy is much better tolerated in the elderly arthritic patient than the double contrast barium meal, as patients can lie comfortably under light sedation rather than be subjected to extensive maneuvering on the $\mathrm{x}$-ray table.

While there is a general acceptance that NSAIDs can cause damage to the gastroduodenal mucosa, the extent of this risk has been controversial (1). Most studies indicate a relative risk for the prevalence of NSAID-associated gastric ulcer of 46 times the risk of the general population, and for duodenal ulcer 8 times (2).

Since the risk of developing a gastric ulcer is considerably greater than for duodenal ulcer, a causal relationship has been suggested between NSAIDs and gastric ulceration. In the case of duodenal ulcer, NSAIDs appear to be associated with the exacerbation of a pre-existing ulcer diathesis.

The presence of an ulcer is associated with a significant increase in the risk of complications. The relative risk of complications ranges from 1.4 to 4.7 in the most reliable studies. This risk is clearly related to increasing age, and epidemiological evidence suggests as much as a quadrupling 
of the risk of complications or death in patients taking NSAIDs (3). The most recent evidence suggests that the risk of a significant gastrointestinal bleed in an individual taking an NSAID is between 5 and $6 \%$ by the end of one year of treatment.

It is thus important to ascertain a history of NSAID treatment before initiating treatment of symptoms of peptic ulcer. But how should this be undertaken?

Patients who develop an NSAID-associated ulcer should have the NSAID stopped, if possible. For those without joint inflammation, analgesics provide a safer alternative. For patients with persisting inflammatory disease it may be possible to reduce the dose, or to consider using nonacetylated salicylates or perhaps low dose corticosteroids, although these should not be combined with NSAIDs.

Treatment of the ulcer can be undertaken with a variety of ulcer therapies. Full dose $\mathrm{H}_{2}$ receptor antagonists or misoprostol can be used to treat gastric ulcer, and a recent study has suggested rapid healing in a subset of prepyloric and gastric ulcer patients continuing to take NSAIDs and treated with omeprazole (4).

For NSAID-associated duodenal ulcer, $\mathrm{H}_{2}$ receptor antagonists and sucralfate have been shown to heal the ulcer whether or not the NSAID is continued. The results of trials with misoprostol in this situation will shortly be available.

However, it is the discrepancy between results for gastric and duodenal ulcer in trials of prophylaxis which confound rational prescription. For example, data currently show that the PGE analogue misoprostol significantly prevents the development of gastric ulceration in arthritis patients taking NSAIDs, and that the $\mathrm{H}_{2}$ receptor antagonist, ranitidine, prevents duodenal but not gastric ulceration. It is important to point out that while these agents have been shown to prevent gastric and duodenal ulcers, respectively, neither has yet been shown to prevent complications. It is reasonable to expect, however, that if the incidence of NSAID-associated ulcers can be significantly reduced, complications may be prevented.

How should we translate this information to clinical practice? Since the overall risk of complications is about one in 5500 NSAID prescriptions, and there were 10.7 million prescriptions for NSAIDs in Canada in 1989, the costs of co-therapy would be considerable, and prophylaxis is clearly not appropriate for all patients taking an NSAID. Prophylaxis should be considered, however, for patients with a previous history of peptic ulcer, especially with complications; any elderly patient whose general medical condition could not tolerate an ulcer complication; and patients who continue to smoke cigarettes and are thus at significantly greater risk of ulcer recurrence.
If the location of a previous ulcer is known; misoprostol should be chosen for a gastric ulcer and an $\mathrm{H}_{2}$ receptor antagonist for a duodenal ulcer. If the site of previous ulcer is not known, it could be argued that since the risk of gastric ulcer is significantly greater while taking NSAIDs that misoprostol should be given. About one-half of all NSAIDassociated complications occur, however, with duodenal ulcers and one might consider an $\mathrm{H}_{2}$ receptor antagonist appropriate. Since there are no clear studies to guide us in this instance, either drug would seem an appropriate choice until further evidence becomes available.

Many of these controversial issues are addressed more fully and eloquently in the contributions to this symposium. They serve to highlight the extent of the research which has been undertaken in this perplexing condition, but more importantly, they point the way to further important studies which are necessary for better management of this problem in the future. For example, how should patients at risk be identified? Are there risk factors other than age? Is the sex of the patient important or do more women seem to get this problem simply because more are treated? Are patients who are selected for prophylactic treatment on the basis of previous ulcer or symptoms any different from those who present with complications? While short term, three month studies show that treatments can reduce the incidence of NSAID-associated ulcer, will they predictably reduce complications? Finally, what are the real costs of prophylaxis? These and many other questions are likely to be answered in the near future, and the recommendations made here and at the symposium may well change as our knowledge evolves. For the time being, however, the conclusions of this symposium should serve as a valuable guide.

\section{REFERENCES}

1. Bijlsma JWJ. Treatment of endoscopy-negative

NSAID-induced upper gastrointestinal symptoms with cimetidine: An international multicentre collaborative study. Aliment Pharmacol Ther 1988;51:75-84.

2. Caldwell JR, Roth SH, Wu WL, et al. Sucralfate treatment of non-steroidal anti-inflammatory drug-induced gastrointestinal symptoms and mucosal damage.

Am J Med 1987;83(Suppl 38):74-82.

3. Hawkey CJ. Non-steroidal anti-inflammatory drugs and peptic ulcer. Br Med J 1990;300:278-83.

4. McCarthy DM. Non-steroidal anti-inflammatory drug-induced ulcers: Management by traditional therapies. Gastroenterology 1989;96:662-74.

5. Langman MJS. Epidemiologic evidence on the association between peptic ulceration and anti-inflammatory drug use. Gastroenterology 1989;96:640-6.

6. Walan A, Gader JP, Classen M, et al. Effect of omeprazole and ranitidine on ulcer healing and relapse rates in patients with benign gastric ulcer. N Engl J Med 1989;320:69-75. 


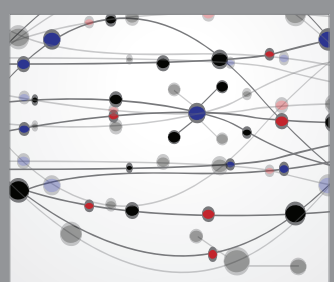

The Scientific World Journal
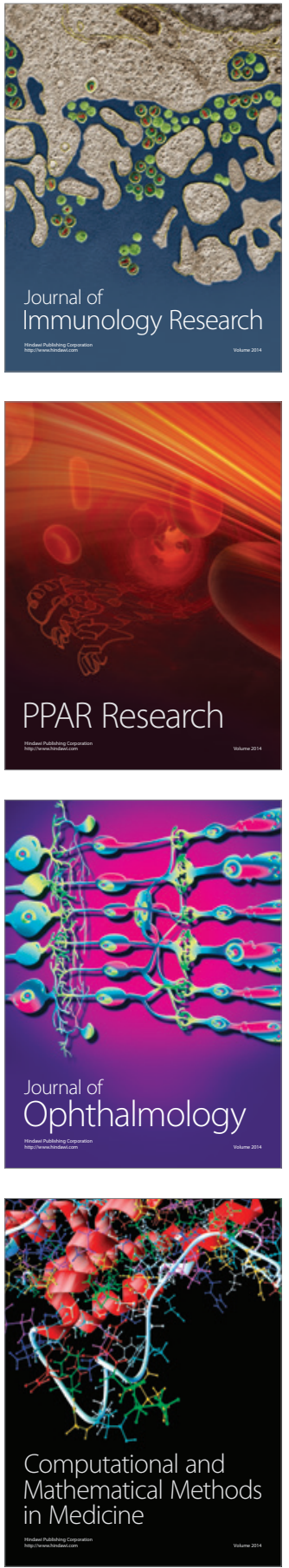

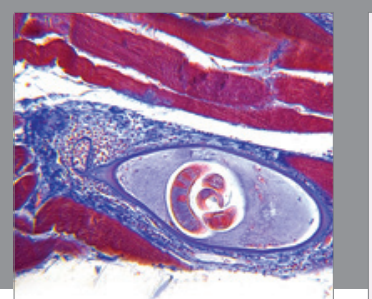

Gastroenterology Research and Practice

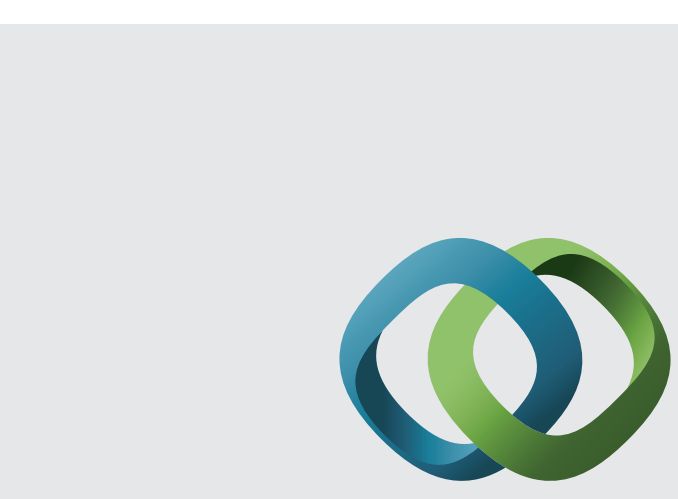

\section{Hindawi}

Submit your manuscripts at

http://www.hindawi.com
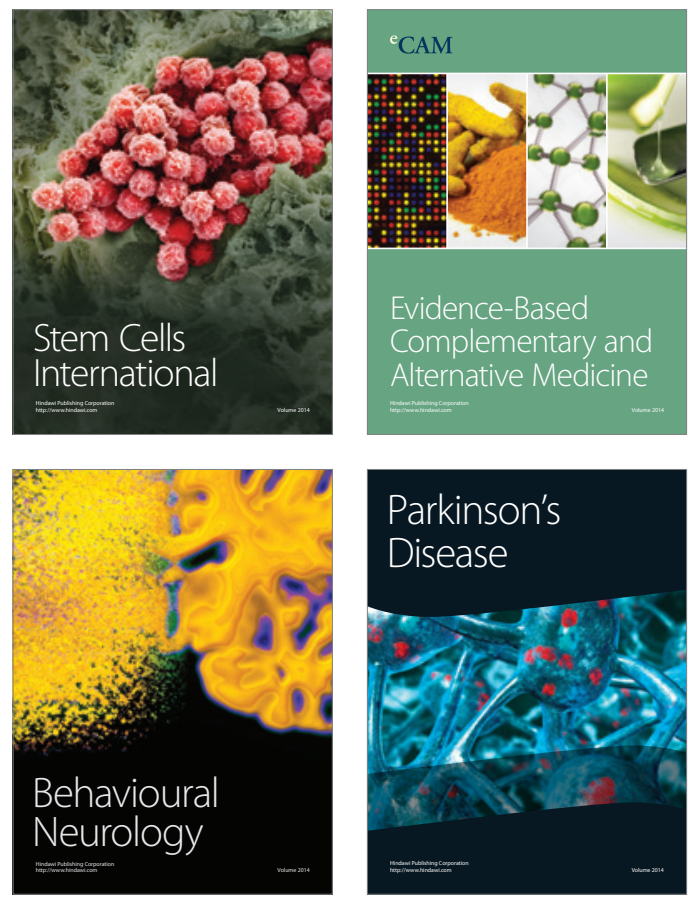
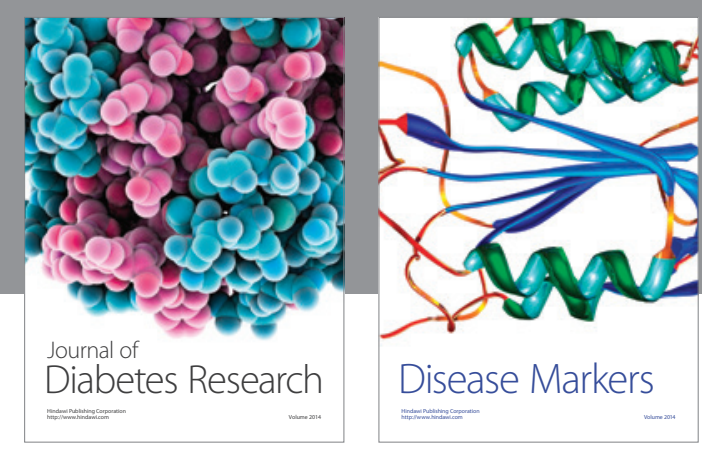

Disease Markers
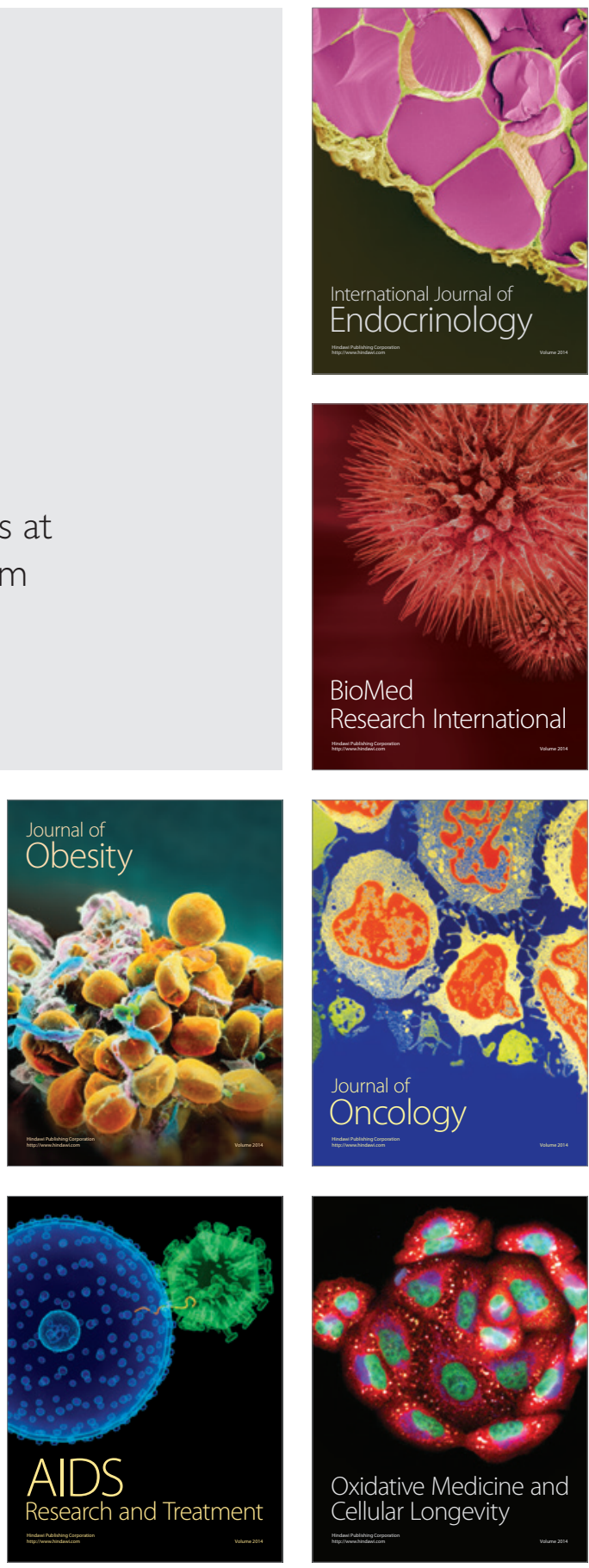